\title{
Reconstituting Phenomena
}

\author{
Maria Kronfeldner
}

in U. Mäki et al. (eds.), Recent Developments in the Philosophy of Science: EPSA13 Helsinki, European Studies in Philosophy of Science 1, DOI 10.1007/978-3-319-23015-3_13

\begin{abstract}
In the face of causal complexity, scientists reconstitute phenomena in order to arrive at a more simplified and partial picture that ignores most of the "bigger picture." This paper will distinguish between two modes of reconstituting phenomena: one moving down to a level of greater decomposition (toward organizational parts of the original phenomenon), and one moving up to a level of greater abstraction (toward different differences regarding the phenomenon). The first aim of the paper is to illustrate that phenomena are moving targets, i.e., they are not fixed once and for all, but are adapted, if necessary, on the basis of the preferred perspective adopted for pragmatic reasons. The second aim is to analyze in detail the moving-up mode of reconstituting phenomena. This includes an exposition of the kind of pragmatic-pluralistic picture resulting from it.
\end{abstract}

Keywords: reconstituting phenomena, causal complexity, abstraction, disciplinary perspectives, pluralism, nature-nurture. 


\section{Causal complexity and the trick of the archer}

When many causal factors are involved in the production of a phenotypic trait of an organism, then life scientists call these traits causally complex. If the focus is on genetic factors, then such traits are said to be polygenic (rather than monogenic); if environmental factors are also part of the story, then the traits are called multifactorial or even multilevel.

Yet, as a matter of fact, many explanations of such causally complex traits use simplifying strategies in the sense that they ignore most of the respective causal factors. This has led to many of the pernicious nature-nurture wars of the $20^{\text {th }}$ century with some scientists ignoring genetic factors (nature), and others environmental ones (nurture).

The kind of causal complexity at issue is compatible with the trait itself being of a rather simple nature. Even a trait such as body height - which is simple in the sense that it has no parts and is easy to define and measure (in contrast to a trait such as intelligence or schizophrenia) - is (as far as we know) causally complex. There is not one gene for body height, but lots of genes influencing the trait, each with a tiny effect, and each interacting with lots of other causal factors, including environmental ones (see Visscher 2008). The kind of complexity that is at issue in this paper is thus a many-to-one causal complexity. ${ }^{1}$

In principle, we can react to causal complexity of that sort with two simplifying strategies (used intentionally or nonintentionally): (a) by selectively focusing on particular causes, while relegating other causally relevant factors to the status of mere conditions (causal selection); and (b) by dividing the phenomenon that we want to explain into parts or states that are more tractable (reconstituting phenomena). ${ }^{2}$ These two simplifying strategies conquer complexity by dividing either the explanans or the explanandum, or both. As a result, we get a partial, simplified picture of, ideally, one-to-one relationships: effects that "have a cause of their own" and causes that "have an effect of their own."

This paper focuses on the second simplifying strategy, reconstituting phenomena, even though the first enters the picture. The paper aims to show that, first, phenomena are moving targets and that, second, there is a mode of reconstitution of phenomena that moves up to a level of greater abstraction rather than down to a level of greater decomposition. Only the latter is currently acknowledged in the philosophical literature discussing complexity and causal explanation in the life sciences, e.g., in Bechtel and Richardson

\footnotetext{
1 It can be contrasted with organizational complexity, dynamic complexity, and semantic complexity, the latter, pointing at definitional heterogeneity of the phenomenon and the problems surrounding construct validity.

2 By "phenomena," I simply mean objects of research, i.e., features of the world that we want to describe and explain. Thus observability is not assumed. On the latter, see the classic paper on the distinction between data and phenomena from Bogen and Woodward (1988). For details on identifying phenomena in actual scientific practice, see Feest (2011).
} 
$(1993 / 2000) .^{3}$ Part of the reason for this is a focus on mechanistic explanation, which is widespread but not always what scientists intend. Sometimes, they are interested in less simply in what I will call "difference explanations." A third major aim of the paper is then to derive some consequences regarding the kind of pluralism entailed when scientists reconstitute (i.e., reconstruct) phenomena by moving up to a level of greater abstraction.

Before I proceed to examples of the two modes of reconstituting phenomena, I want to illustrate my first aim of the paper by referring to a Jewish parable, the parable of the king and the archer: There was once a king who was in urgent need of a very good archer. After desperately searching for a time, he discovered a field strewn with bull's-eyes. Inquiring about the archer, he was directed to the local library, where he found Harold reading a book through thick glasses. The king was skeptical and asked Harold how he managed to be such a good archer. Harold replied: "Sir, it is really very simple. I stand in front of the fence and shoot my arrow. Then I paint the target around it, with the arrow at the exact center" (quoted by Schwartz 1998).

Scientists often use the archer's trick: they choose one convenient, easy-to-handle causal factor (e.g., bits of DNA) as an arrow (causal selection), shoot it (i.e., experiment with it or collect data about it), and then, if necessary, adapt their phenomena (explananda) afterward (reconstituting phenomena). Phenomena - the scientist's bull's-eyes - are moving targets that are not fixed once and for all, but are adapted. What is fixed (if at all) is the causal factor as the focus of scientific interest, which itself varies according to disciplinary affiliation. This adaptation of the phenomena, furthermore, does not always involve moving down to a level of greater decomposition and therefore toward greater proximity of causal relations, i.e., more details. "More detail" is not always what scientists want. Indeed, they might well prefer to move up to a level of greater abstraction in order to get rid of those causal factors they want to ignore (given their focus of interest).

In Sections 2 and 3, I will illustrate the two modes of reconstituting phenomena. In Section 4, I will explicate in detail the kind of pragmatic pluralism of causal perspectives that results from the simplifying, complexity-reducing strategy of reconstituting phenomena by moving up to a level of greater abstraction.

\section{Reconstituting phenomena by moving down to a level of greater decomposition}

To reduce the complexity of causal relations involved in explaining phenotypic traits, scientists often move their explanandum phenomenon down to a level of greater decomposition, i.e., toward greater proximity and increasingly specific one-to-one causal

\footnotetext{
${ }^{3}$ From whom I take the term "reconstituting phenomena."
} 
relations. Such a move down to a level of greater decomposition happened, for instance, when scientists adapted their explanandum from the Mendelian picture of "one gene explains one phenotype" to the more modest "one gene explains one enzyme" paradigm. The enzyme is an organizational part of the phenotype and consequently located at a level of greater decomposition than the respective phenotype; the enzyme is also a more proximate effect of the gene that has the phenotype as a distal effect.

A more contemporary case of this mode of reconstituting phenomena by moving down to a level of greater decomposition, i.e., toward more proximate effects, is the concept of endophenotypes. An explanandum (e.g., the phenotypic trait schizophrenia) gets partitioned into parts, the endophenotypes (e.g., sensory motor gating, oculumotor function, etc.) (Gottesman and Gould 2003). These are more proximate effects of the genes, and the phenotype results from - and is also, in that sense, composed of - them, even though these "parts" are not organizational (mereological) parts of schizophrenia. Furthermore, even though the move to endophenotypes is a move towards parts of the phenotype, it does not involve a new kind of explanandum, a new level of abstraction, since endophenotypes are the same kind of things as phenotypes and constitute the latter. Yet, the phenomenon (the phenotype as explanandum) is adapted - reconstituted - at a "deeper" level of decomposition.

If scientists remained at the level of the phenotype, despite tremendous causal complexity, then - in the final instance - everything would be connected with everything. But "[i]n pointing at everything, geneticists would point at nothing" as David Goldstein (2009: 1696) recently said. ${ }^{4}$ That is why they adapt their explanandum by moving down to a level of greater decomposition, which thereby gives them increasingly proximate and usually also increasingly specific one-to-one causal relations.

Yet scientists can also move up to a level of greater abstraction, which is a move to a new kind of explanandum phenomenon. In the example I shall use, which is again connected to the phenogenesis of traits, it is a move from explaining traits to explaining trait differences.

\section{Reconstituting phenomena by moving up to a level of greater abstraction}

To avoid semantic issues about construct validity (how a phenomenon such as schizophrenia can be defined and measured), I use as an example the 'simple' trait that we mentioned above, namely, body height. Figure 1 displays how one can measure the influence of nature and nurture on such a trait, namely, by plotting norms of reactions that ignore all kinds of causal factors except two, a genetic and an environmental one.

\footnotetext{
4 Thanks to Ken Schaffner, who provided me with this nice quote.
} 


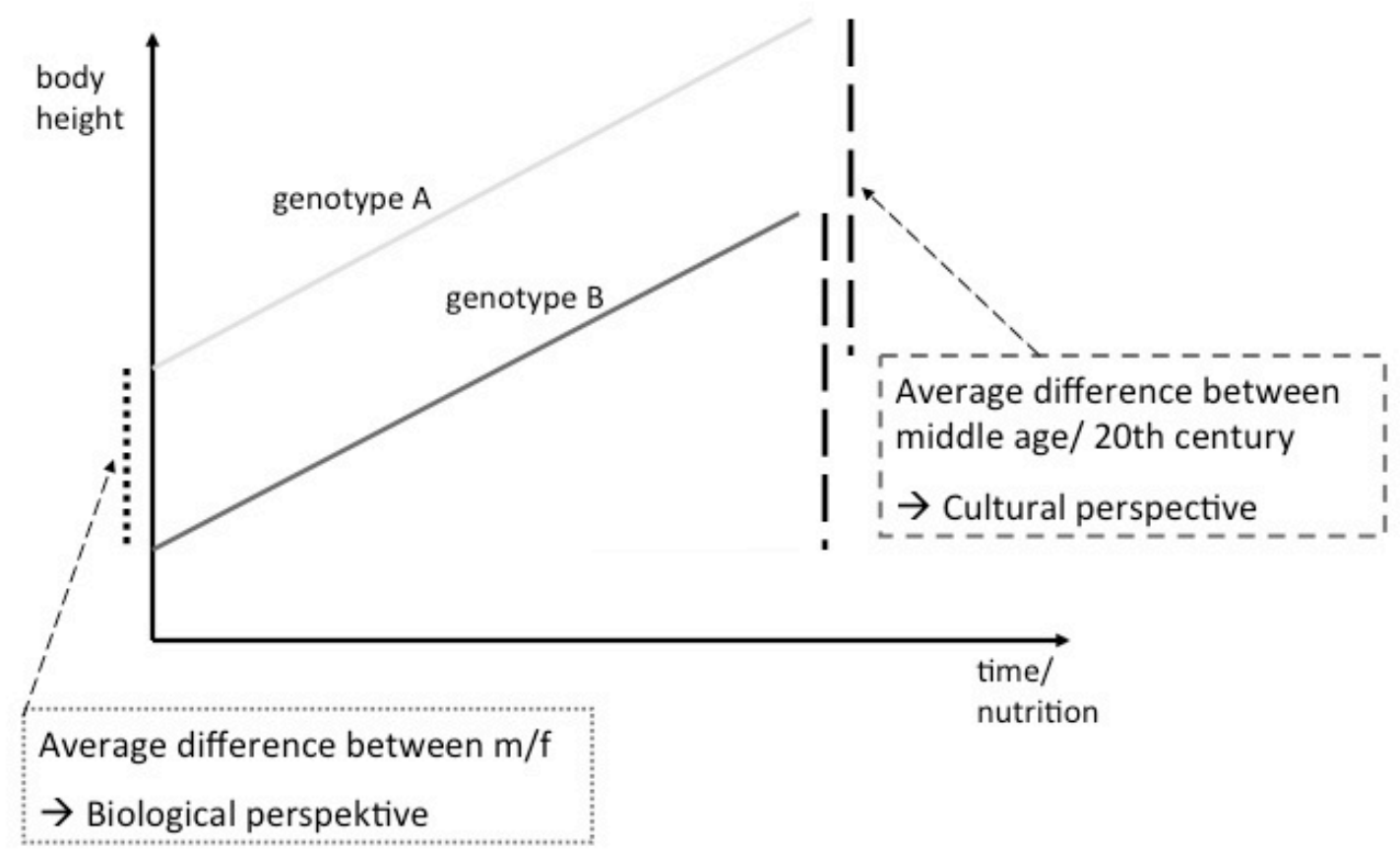

Figure 1: Hypothetical reaction norm for human body height.

At issue is human body height. The displayed norms of reactions (the two grey lines) of the two genotypes (A and B) are hypothetical. Norms of reactions represent the norms of how the two genotypes at issue "behave" regarding a specific trait (e.g., body height) and given a change in environment (e.g., better nutrition over time, the period from the $15^{\text {th }}$ to the $21^{\text {st }}$ century).

The explanations based on such causal analysis are not mechanistic explanations in the narrow sense of explaining how the trait emerges. The explanations are focused on why, or due to which causes, the trait emerges, even if the developmental mechanisms are blackboxed. The explanations involve causes as difference makers. ${ }^{5}$ Figure 1 clearly shows that nature and nurture causally interact to bring about a trait: changing the environment makes a difference to height as well as changing the genotype. Nature and nurture are both causally relevant.

Nonetheless, some theorists still want to give priority to nature. Francis Fukuyama, for instance, in Our Posthuman Future (2002), admits that human body height has increased

\footnotetext{
5 See Woodward (2003) as one way to spell out what difference making can mean.
} 
over the centuries, compared to our "Middle Age" ancestors in the 15 th century, due to improved nutrition. Fukuyama thus joins the chorus of the interactionist consensus and concedes that, yes, of course, body height is due to nature and nurture. A few lines later, however, he states that "the average male-female differences are the products of heredity, and thus nature" (130-3). Here, he is talking about "products of heredity," i.e., he is using causal language. Is he contradicting himself? No, he is simply performing the rhetorical sleight of hand that Keller already criticized in her 2010 book, a sleight of hand that I call implicitly changing the explanandum, namely, a move from explaining traits to explaining trait differences. ${ }^{6}$ What Fukuyama says is that, yes, certainly, changing the environment makes a difference to height, the trait, but it does not make a difference to the difference between (male and female average) heights. (The example is certainly an idealized case (see below for discussion). The hypothetical average difference between male and female body height caused by the gene is depicted by the dotted vertical line on the left-hand side of Figure 1 . Obviously, this difference is of interest to geneticists, simply on account of their disciplinary affiliation. They want to know whether a gene makes a difference. That's their job. They therefore look for the difference the gene makes a difference to, and ignore the rest. Others have a different job. A cultural anthropologist, for example, might reply: "Hey, that is hereditarianism and wrong, since we agreed that nutrition makes a difference too, didn't we?" - "Well, we did, but making a difference to what? It makes a difference to height, but it does not make a difference to the difference between males and females," the biologist replies, pointing to the dotted vertical line on the left-hand side of Figure 1, which stays the same whatever you do with the depicted environmental variable. The cultural anthropologist answers: "Ah, I see, right; but wait a minute, the environment also makes a difference to a difference, but to a different difference: it makes a difference to what's represented by the dashed vertical line in Figure 1 (on the right-hand side), namely, the difference in (average) height (of humans altogether) between the Middle Ages and today. This is the difference that interests me (as a historian or cultural anthropologist). It is a difference to which your genetic factor is not making any difference."

Our imaginary dialog between Fukuyama and a cultural anthropologist can also be put in terms of different contrasts to the explanandum. While some (e.g., Fukuyama) are interested in why men and women have (on average) different body heights (rather than the same), others are interested in why humans (altogether, on average) have a different height today (compared to 500 years ago).

\footnotetext{
${ }^{6}$ I take the distinction between traits and trait differences as explananda from Lisa Gannett (1999).
} 
The move from traits to trait differences (as explananda) amounts to an immunization against either biological or cultural perspectives. We mentioned Fukuyama as representing the one camp (being interested in genes only), and there is, for example, Alfred L. Kroeber, champion of cultural determinism, representing the other. In order to explain culture, Kroeber believed, we do not need any reference to nature, i.e., genes, etc. He did not claim that there are no biological factors causally relevant for development of human characteristics, but he defended the right to ignore all biological causal factors for the cultural patterns - differences between humans in time or place - that he was interested in. 7 There are various reasons for wishing to show the causal irrelevance of the environment (or of genes), and thereby claiming the right to ignore it. These might be reasons of academic discipline (after all, as mentioned, it is the geneticist's job to be interested in the causal relevance of genes) or political reasons (i.e., interests in maintaining the status quo and therefore downplaying the significance of certain factors in a causal explanation, e.g., poverty). Yet, in each case, the logic of the simplifying strategy stays the same.

So, in the end, we have two perspectives, a biological and a cultural one, with different differences as respective explananda. Those espousing these different perspectives (involving different differences and consequently different difference makers) are unable to talk to each other, since each side now has a "cause of its own" and a cause with an "effect of its own," a one-to-one monocausal explanation, despite the fact that in the world everything interacts.

The Jewish parable about the king and the archer illustrated nicely what happens when you start with a disciplinary bias toward a specific cause. Take Fukuyama again: he shoots his arrow (the cause he is interested in) and paints his explanandum accordingly. This also works the other way round: you can start with a difference you are interested in (the target) and then shoot your arrow. As the parable illustrated, the latter is more difficult. Given this, a follow-up historical study (that cannot be provided here) might show that therefore many scientific fields got historically increasingly organized around preferred kinds of causal factors (and the methods suitable to study them) rather than directly around specific phenomena.

To recapitulate: scientists can adapt an explanandum (i.e., paint a target around the point to which the preferred causal factor has been shot like an arrow, using the methods available for the specific scientific field). They can do so by moving down to a level of greater decomposition, but also by moving up to a level of greater abstraction, from a trait (a property of an organism) to statistical trait differences between organisms (i.e., to the difference between averaged values of a trait constructed as a variable). This is done in order to simplify the

7 See, as a very explicit and late statement on this, Kroeber (1952: 7). For details on the emergence of his cultural determinism at the beginning of the 20th century, see Kronfeldner (2009). 
causal complexity (many causes) at hand, i.e., in order to arrive at a partial picture that gives priority to a preferred cause of interest, even though in the world everything interacts.

\section{The pragmatic pluralism that results from moving up to a level of greater abstraction}

The moving-up mode of reconstituting phenomena is important in that it helps us understand how pluralism regarding causal perspectives arises, especially regarding naturenurture debates. It also helps us understand how scientists manage to give partial explanations despite causal complexity and independently of mechanistic explanations of the developmental processes involved. Eight points are important in order to avoid misunderstanding the pragmatic-pluralistic picture resulting from this analysis.

(1) An idealized case. The hypothetical norm of reaction of height that I used in the example is certainly a simple and (even more importantly) an idealized case: only if the lines run in parallel, does nurture not make a difference to the average difference between male and female body height. The idealization is intentionally used here, since the point I want to make is that people like Fukuyama take the idealization as a guide. In non-idealized cases, it is not sufficient to adapt the explanandum to give one's own causal factor a special, exclusive status. Yet the causal influence in which one is not interested can still be successfully downplayed, either by ignoring it completely, or by idealizing it away (assuming it to be randomized or randomizing it), or by abstracting from it (determining it as fixed). Although the "due to nature" is then less exclusive, the move from a trait to a trait difference as explanandum is still the first and necessary step toward further simplifications. If the interaction between genes and environment is nonlinear, the simplifying strategy has (so to speak) "belied" us - as Cartwright's (1983) laws of physics do - but not in an impeding manner. It is an idealization and defines a heuristic, a research strategy, in order to arrive at some partial knowledge about the genesis of phenotypic traits. The idealized norm of reaction is intended as a simple example to illustrate the abstraction process in such cases. It shows how two different preferred causes or two different choices of differences (which are phenomena in their own right and thus explananda) place different things into the foreground or background. It thus helps to make sense of the fact that scientists ignore some causal factors, i.e., parts of the causal complexity, pertaining to the development of phenotypic traits.

(2) It is a change in the kind of explanandum. If there is the described change in kind of explanandum, then this is more than a change from a trait token to a trait as a type. It is a change in the kind of explanandum (from traits to trait differences). If it were only a change from a trait token as explanandum (individual body height) to a trait type as explanandum 
(body height in that population), then we would simply move from explaining a token to explaining the typical development of the trait in a type, which would still be explaining similarity, rather than difference. In order to explain typical development of body height, you would also need nature and nurture interacting in order to give an adequate explanation, even in the idealized case of parallel norms of reactions. This is not necessarily the case if you explain averaged differences of height.

(3) Difference explanations and trait explanations. Even though different kinds of explanandum are established, the kind of explanation is the same, e.g., explanations involving difference makers. I take Keller (2010) to assume that with a change from traits to trait differences as explanandum, we also change the kind of explanation, because we change from causes as trait makers to causes as difference makers. Trait explanations and difference explanations would then be two categorically different kinds of explanation. Yet trait makers are simply a set of difference makers, namely, that very set that gives us (in the final instance) the total cause, in the sense of all the contributing causes that together are (in a given causal structure, with all its background conditions) sufficient for the to-be-explained phenomenon. If you ignore culture in a trait explanation, then you cite only a few of the many actual difference makers. If you ignore nature in a trait explanation, you do the same. What you ignore is always of the same kind: you ignore certain difference makers.

Furthermore, the question as to whether you are interested in a trait explanation or in a difference explanation depends on your pragmatic (or even political) goals: if the goal is, for instance, to produce something (e.g., that an enemy contracts malaria), we indeed need more than knowledge about one or only a few difference makers. We need knowledge about the total, complete cause (a set of causes, actually). If we want to prevent malaria, however, we do not need such comprehensive complete knowledge of the causes. Knowledge of one of the difference makers (e.g., a mosquito bite) is enough to explain the occurrence of malaria as long as that difference maker can be regarded as a necessary condition in the context at issue (i.e., as something without which the disease would not have occurred in the context).

Thus, irrespective of whether we have a trait explanation or a difference explanation, both refer to difference makers relevant to the respective explanandum, given the pragmatic goal at hand (e.g., production, prevention, prediction, etc.). Keller is therefore right that an explanation that invokes only one difference maker is not the same as an explanation that gives you the total cause, a set of difference makers. But the difference is a difference in degree only - a question of how many difference makers you cite: one, two, three, many (or, all, which will usually be beyond our capacities anyway). How many you need for an adequate explanation depends on your pragmatic goals and on the differences (to which something makes a difference) into which you partition the phenomenon. 
(4) Parity at the level of perspectives. When biologists and cultural anthropologists, given their different interests, fight about the contribution of nature-nurture to a trait such as body height, then this can be seen as "talking at cross purposes": if they disagree about the explanandum, which they do when they settle for a division of labor along the lines of the different differences in which they are interested, then the explanations they respectively espouse are neither competing alternatives nor direct complements. The resulting explanations are rather alternative ways to reconstitute the original phenomenon toward different differences that do not compete with each other but can be combined later via the shared relationship of the reconstituted phenomena to the original phenomenon. There is thus a pragmaticpluralistic parity and complementarity between the parties at the level of the perspectives used to study the different differences regarding a trait.

(5) Partial, but fruitful. If you ignore culture by changing from trait to trait differences, as Fukuyama did, you can neutralize the influence of the environmental factor by tailoring your difference. It's a trick, but it works. The knowledge generated by the different perspectives elucidates the different differences (e.g., the difference in height between the Middle Ages and now, and the alleged difference between males and females). Although the result here for each perspective is partial knowledge, it is still a fruitful strategy to reconstitute phenomena by moving up to a level of greater abstraction. It is plausible that the division of labor we have in science, which has accelerated tremendously in the last 200 years, is itself, in part, the historical effect of the complexity of a world that we are only gradually discovering. On the way towards more of it, we can thus consider it a good thing that there are different kinds of experts devoted to their "own" kind of causes, so that there is at least some knowledge, even if it is partial (i.e., from studying one factor in isolation, while the others are ignored or held fixed or randomized).

(6) Objectivity and differences worth studying. The partial perspectives are certainly biased. Consequently, objectivity can only be reached by including them all, as partial but legitimate perspectives, as Longino (2013) illustrates in detail with respect to nature-nurture debates. Yet, in the final analysis, it all depends on whether the respective differences are worth studying since we can most likely slice up any phenomenon into as many differences as there are theoretical perspectives in science. Since there is no purely scientific way to argue that any of the differences is more important than the other, social values will have an influence on that. For instance, as Kourany (draft) stresses regarding cognitive difference research, a society might well decide that certain differences (e.g. between races or gender) should not be studied anymore, for social reasons.

(7) Integration at the level of traits. The integration of the partial difference explanations on the basis of the described division of labor most certainly occurs at the level of the trait 
that has been fragmented into all kinds of differences regarding the trait. In the long run, a division of labor and integration of the partial causal knowledge are both required for the increase of our knowledge about phenotypic traits. It takes two to tango.

(8) But can the different difference explanations be integrated? A radical pluralism, such as advocated by Longino (2013), denying the possibility of integration of the diverging perspectives in the nature-nurture debates, is inadequate. It is a pragmatic, integrative pluralism that is advocated here. Integration of the causal knowledge arrived at by looking at causal factors in isolation is possible, as any norm of reaction exemplifies. We can combine the knowledge that a specific genotype makes a difference with regard to a difference in body height (in the measured situation) and the knowledge that nutrition influences a different difference regarding body height (with the same limitations), even in non-idealized, i.e., non-linear cases. We might even learn something about further patterns of dependence, as illustrated in the gene-interaction cases studied by Caspi et al. (e.g., 2002) or as described by Kitcher (2001).

Longino (2013), however, demands that true integration be not only an integration of knowledge that the respective factors make a difference but also an integration of knowledge about how much difference these make. She assumes that approaches involved in naturenurture debates always want an answer to a "how much" question, which I think is a mischaracterization. In my opinion, she mistakenly transfers the 'how much' approach, as used in quantitative behavioral genetics, to other disciplines for the study of humans. Such a quantitative integration is indeed impossible, as the now classic discussion on apportioning causality with respect to nature-nurture shows. ${ }^{8}$ Yet few scientists, I would say, care for it as a goal (except certainly traditional quantitative behavioral geneticists). Furthermore, true integration for Longino is only happening if all the partial knowledge derived from different perspectives is combined into a "single comprehensive account" (Longino, personal communication, Dec 2014). ${ }^{9}$ This sets the standards so high so that integration is - simply because of that standard of complete knowledge - usually impossible since it is usually beyond human capacities to give complete causal accounts. Finally, for the social aims at issue in nature-nurture debates, "how much" questions and a completeness standard are not important either, given that interventionist social policies can operate with less than "how much" knowledge integrated into a complete account of the phenogenesis of the trait.

\footnotetext{
8 J. St. Mill (1858) already acknowledged the problem under the label of a "composition of causes." See, for instance, Sober (1994), Keller (2010), and Walsh (2013) on this classic problem of apportioning "how much" in nature-nurture debates.

${ }^{9}$ Compare Tabery, Preda and Longino (2014), where Longino stresses in reply to Tabery that anything short of a "complete and comprehensive" account of the mechanisms involved in the phenogenesis of a trait is not adequate.
} 
From my point of view, Longino's picture demands too much and - as a result - is too pessimistic, as if there were no way to deal with uncertainty regarding "how much" nature and nurture contribute to a trait. In the final instance, integrating difference explanations should elucidate how a trait such as body height is produced mechanistically (i.e., stepwise), without being able to say how much each factor contributes in relation to each other, i.e., comparatively, and however partial that mechanistic explanation is. In sum, the above-mentioned nonquantitative partial integration - of knowledge that certain factors make a difference (to different differences) regarding a trait - can contribute to mechanistic explanation, even though the difference explanations are themselves not necessarily mechanistic. ${ }^{10}$

My perspective on integration is thus similar to Sandra Mitchell's (2003) integrative pluralism. However, since Mitchell is not concerned with nature-nurture issues, she seems to ignore that there are cases of partial causal perspectives where we can partition the explanandum and divide it so that it can be shared (being related to the same trait), without having the exact same explanandum (by being focused on different differences). In her integrative pluralism (as in Longino's nonintegrative pluralism), there seem to be different questions and different causes (derived from incongruent different perspectives), but only one trait and no trait differences mediating the integration of the different causes.

Keller (2010), by contrast, falls short in the exact opposite direction, by situating difference explanations and trait explanations too far apart, albeit while acknowledging the distinction between a trait and trait differences as explanandum.

\section{Summary}

This paper addressed the epistemology of simplifying explanatory strategies. It focused on whether we can make sense of the fact that scientists ignore some causal factors involved in the explanation of a phenomenon by reconstituting their explanandum phenomenon (i.e., by redefining it so that it better fits the aims with which one started). The answer is that, as a pragmatic-pluralist, we can. This paper also explicated how disciplinary boundary politics enter the business of reconstituting phenomena: disciplinary perspectives constitute the "arrows" (i.e., the causal factors at reach or preferred from that perspective) that are "shot" and according to which general and shared explananda (i.e., complex traits such as body height, schizophrenia, etc.) are adapted so that they can be "bull's-eyes" (i.e., explananda that are less causally complex and fitting to the arrows shot). Depending on case, the

10 See Tabery (2009) for more on integrating mechanistic explanation and the search for differences. See also the exchange in Tabery, Preda and Longino (2014). 
reconstitution of phenomena will be down a level of composition (e.g., in the case of the concept of endophenotypes) or up a level of abstraction (e.g., in the case of explaining different differences).

\section{Acknowledgements}

I want to thank the Center for Philosophy of Science at the University of Pittsburgh and Martin Carrier for their great support during the time this paper was written. I also want to thank Bill Bechtel, Uljana Feest, Lisa Gannett, Peter Godfrey-Smith, Jens Harbecke, Evelyn Fox Keller, Helen Longino, Sandra Mitchell, Ken Schaffner and one of the two anonymous referees for interesting and helpful feedback. I want to particularly thank Alexander Reutlinger for the many inspiring discussions related to the topics of this paper. 


\section{References}

Bechtel, W., \& Richardson, R. C. (1993/2000). Discovering complexity: decomposition and localization as strategies in scientific research. Princeton, NJ: Princeton University Press.

Bogen, J. Woodward, J. (1988). Saving the Phenomena. The Philosophical Review, 97, 303-52.

Cartwright, N. (1983). How the laws of physics lie. Oxford: Clarendon Press.

Caspi, A., McClay, J., Moffitt, T. E., Mill, J., Martin, J., Craig, I. W., et al. (2002). Role of genotype in the cycle of violence in maltreated children. Science, 297, 851-854.

Feest, U. (2011). What exactly is stabilized when phenomena are stabilized? Synthese, 182, 57-71.

Fukuyama, F. (2002). Our posthuman future. New York: Farrar, Straus and Giroux.

Gannett, L. (1999). What's in a cause?: The pragmatic dimensions of genetic explanations. Biology and Philosophy, 14, 349-373.

Goldstein, D. B. (2009). Common genetic variation and human traits. New England Journal of Medicine, 360, 1696-1968.

Gottesman, I. I., \& Gould, T. D. (2003). The endophenotype concept in psychiatry: etymology and strategic intentions. The American journal of psychiatry, 160, 636-645.

Keller, E. F. (2010). The mirage of a space between nature and nurture. Durham, NC: Duke University Press.

Kitcher, P. (2001). Battling the undead: How and (how not) to resist genetic determinism. In R. Singh, C. Krimbas, D. Paul, \& J. Beatty (Eds.), Thinking About Evolution: Historical, Philosophical and Political Perspectives (Vol. 2, pp. 396-414). Cambridge: Cambridge University Press.

Kroeber, A. L. (1952). The nature of culture. Chicago: The University of Chicago Press.

Kronfeldner, M. (2009). If there is nothing beyond the organic... : Heredity and culture at the boundaries of anthropology in the work of Alfred L. Kroeber. NTM - Journal of the History of Science, Technology and Medicine, 17, 107-133.

Kourany, J. (draft). Should some knowledge be forbidden? The case of cognitive differences research. Presented at the Fishbein Workshop in the History of the Human Sciences, Chicago, Feb 21, 2014.

Longino, H. E. (2013). Studying human behavior : how scientists investigate aggression and sexuality. Chicago: The University of Chicago Press.

Mill, J. S. (1858). A system of logic, ratiocinative and inductive. New York: Harper \& Bros.

Mitchell, S. D. (2003). Biological complexity and integrative pluralism. Cambridge: Cambridge University Press.

Schwartz, S. (1998). The role of values in the nature/nurture debate about psychiatric disorders. Social Psychiatry and Psychiatric Epidemiology, 33, 356-362.

Sober, E. (1994). Apportioning causal responsibility. In From a biological point of view: Essays in evolutionary philosophy (pp. 184-200). Cambridge: Cambridge University Press.

Tabery, J. (2009). Difference mechanisms: explaining variation with mechanisms. Biology and Philosophy, 24, 645-664.

Tabery, J., Preda, A. \& Longino, H. (2014). Pluralism, Social Action and the Causal Space of Human Behavior. Metascience, 23, 443-59.

Visscher, P. M. (2008). Sizing up human height variation. Nature Genetics, 40, 489-490.

Walsh, D. M. (2013). The negotiated organism: inheritance, development, and the method of difference. Biological Journal of the Linnean Society 112: 295-305.

Woodward, J. (2003). Making things happen: A theory of causal explanation. New York: Oxford University Press. 